\title{
Plasmon Resonances in Nanowires with a Non-regular Cross-Section
}

\author{
Olivier J. F. Martin \\ Electromagnetic Fields and Microwave Electronics Laboratory, \\ ETZ-G46, ETH-Zentrum, 8092 Zürich, Switzerland \\ martin@ifh.ee.ethz.ch
}

\begin{abstract}
We investigate numerically the spectrum of plasmon resonances for metallic nanowires with a non-regular cross-section in the $20-50 \mathrm{~nm}$ range. After briefly recalling the physical properties of metals at optical frequencies, we point out the intrinsic difficulties in the computation of the plasmon resonances for nanoparticles with a non-regular shape. We then consider the resonance spectra corresponding to nanowires whose cross-sections form different simplexes. The number of resonances strongly increases when the section symmetry decreases: A cylindrical wire exhibits one resonance, whereas we observe more than 5 distinct resonances for a triangular particle. The spectral range covered by these different resonances becomes very large, giving to the particle specific distinct colors. At the resonance, dramatic field enhancement is observed at the vicinity of non-regular particles, where the field amplitude can reach several hundred times that of the illumination field. This near-field enhancement corresponds to surface enhanced Raman scattering (SERS) enhancement locally in excess of $10^{12}$. The distance dependence of this enhancement is investigated and we show that it depends on the plasmon resonance excited in the particle, i.e. on the illumination wavelength. The average Raman enhancement for molecules distributed on the entire particle surface is also computed and discussed in the context of experiments in which large numbers of molecules are used. Finally we discuss the influence of the model permittivity which enters the calculation, as well as the resonances shift and broadening produced by a water background.
\end{abstract}

\section{Introduction}

The interaction of light with small metal particles has been of great interest for many centuries. Medieval artisans, for example, made use of metal colloidal particles in the production of certain types of stained glass. By tuning the particle size and composition, they were able to produce glasses with specific colors, which were the signatures of the plasmon resonances excited in the particles. Since a plasmon resonance does not wear out, we can still enjoy these shiny colors today. The fact that there is a connection between the scattered spectrum of a nanoparticle and its physical properties was established long ago. For instance, Faraday noted "that a mere variation in the size of its particles gave rise to a variety of colors" [1]. This fact can easily be observed in a solution of colloidal gold or silver under the optical

J. Tominaga and D. P. Tsai (Eds.): Optical Nanotechnologies,

Topics Appl. Phys. 88, 183-210 (2003)

(C) Springer-Verlag Berlin Heidelberg 2003 
microscope: although all particles have a similar composition, they shine with several different colors.

The relation between the shape and the spectrum was recently highlighted in a beautiful experiment by Schatz and co-workers [2]. The transformation of the plasmon-resonance spectrum was correlated to the changes of the form of the silver nanoparticles as they evolved from a spherical shape into a prismlike shape. A similar study was presented by Mock et al., who systematically investigated the effect of size and shape on the spectral response of individual silver nanoparticles [3].

Beyond creating nice color effects, the current interest in colloidal metals is driven by two major topics. On the one hand, there is the phenomenon of surface-enhanced Raman scattering (SERS), wherein the Raman signal of ensembles of molecules adsorbed on rough metal surfaces can be enhanced by a factor of $10^{7}[4,5,6,7]$. In recent experiments, Raman enhancements of up to $10^{12}$ were even reported for single molecules located on so-called "hot spots" $[8,9,10]$. The adsorption of the molecule on the metal can participate in the Raman enhancement $[11,12]$. However, it is believed that the excitation of plasmons in the metallic nanoparticles creates greatly enhanced local electromagnetic fields that contribute the major component of the SERS effect.

On the other hand, a new field of nano-photonics is currently coming to life, where plasmon-resonant nanoparticles are used to guide and switch light at the nanoscale. New classes of waveguides, which utilize plasmon resonances as means of transporting electromagnetic energy have been demonstrated $[13,14,15]$. The utilization of plasmon-resonant nanoparticles can also dramatically reduce the spot size, thereby increasing the data storage density for next-generation optical data storage disks $[16,17]$. Finally, the utilization of plasmon-resonant particles as switchings elements opens exciting perspectives for new active devices [18]. These different active and passive components may pave the way for the all-optical dream: a network where information transmission and switching occur entirely at the optical level.

In order to successfully implement plasmon resonances both in chemistry and in optics, it is mandatory to further our understanding of the underlying physics for nanoparticles with an arbitrary shape. This knowledge should allow us to realize plasmon-resonant particles with tailored properties for specific applications. For example, by tuning the plasmon resonance frequency one could fabricate particles adapted to specific chemical compounds or produce particles with several well-defined resonances, covering a given frequency range. Also, the near-field distribution in the vicinity of the particle could be tuned and specific field distributions which would provide the strongest enhancement at the location of the active molecular site could be developed. Finally, the plasmon resonance linewidth, which is related to the plasmon lifetime - an important parameter for transient and nonlinear phenomena could be tuned by changing the particle properties. 
It should be noted that considerable progress has been made in recent years in the fabrication of metallic nanostructures in a controlled manner, including features in the 10-50 $\mathrm{nm}$ range [19,20,21,22,23,24,25,26,27,28]. Metallic particles with a variety of shapes and dimensions are now readily available for experiments. A thorough understanding of the detailed local fields associated with plasmon-resonant particles is therefore warranted, to enhance the design and optimization of applications based on these particles.

The accurate solution of the local fields of plasmon-resonant particles of arbitrary shape remains, however, a theoretical challenge. Analytical solutions for the fields are known only for particles with a very simple shape, like that of a sphere or an ellipsoid [29,30,31,32,33,34,35,36,37], or spherical shells and periodic cylinder gratings [38,39,40,41,42,43,44]. While electrostatic methods can provide some level of insight [45], complete electromagnetic solutions are needed to obtain accurate results. Many groups have developed methods of solving Maxwell's equations to investigate non-regular plasmon-resonant particles in the $100-200 \mathrm{~nm}$ size range $[46,47,48]$; however, although particles of this size provide a large scattering cross-section (SCS) at the plasmon resonance, the resonances are very broad and the field enhancement in the vicinity of the particles is relatively small.

Over the last few years we have elaborated a numerical approach for the solution of the fields associated with plasmon-resonant nanoparticles of arbitrary two-dimensional geometry, which leads to highly accurate, converged solutions, even for particles having extremely large local enhancement and field variation [49]. With this technique we were able to study some aspects of the plasmon resonances in nanowires with a non-regular cross-section in the sub-100 nm size range $[50,51,52,53]$. Several on-line publications have also illustrated with movies the dynamics of specific effects $[54,55]$. In the present chapter, we shall summarize these results and draw some conclusions for the utilization of plasmon engineering in the nanosciences. We will only consider the case of individual nanowires and not address that of interacting nanowires, where interesting effects can also occur [56,57].

Since our work is theoretical, it appears important to first sketch in Sect. 2 the difficulties associated with the simulation of plasmon-resonant nanoparticles. In Sect. 3 we investigate the relationship between a nanowire cross-section and its plasmon resonance spectrum. An extremely useful tool for understanding the characteristics of each plasmon resonance is its associated polarization charge distribution, as discussed in Sect. 4. Some implications for SERS are discussed in Sect. 5, while the limitation of our Maxwellian approach is addressed in Sect. 6. Conclusions and outlook are given in Sect. 7. 


\section{Model}

\subsection{Metals at Optical Frequencies}

The electromagnetic scattering of metal nanoparticles can be described by solving Maxwell's equations [58,59]. In this model, the complete description of the material properties of the metal is encompassed in the dispersion relation, which gives the complex permittivity $\varepsilon(\omega)$ as a function of the frequency (or wavelength). The dispersion relation measured by Johnson and Christy for silver is shown in Fig. 1 [60]. We shall use these values for our calculations.

Near the plasma frequency $\omega_{\mathrm{p}}$ of the metal, the dispersion relation is governed by the interaction between light and the conduction electron gas, or expressed with their quantum counterparts by the photon-plasmon interaction. The combined exciton is often referred to as plasmon-polariton. For certain metals such as silver, copper and gold, $\omega_{\mathrm{p}}$ is in the visible frequency range. At specific negative permittivity values, plasmon resonances will be excited in these small particles. These specific permittivity values strongly depend on the particle size and shape, since the boundary conditions imposed by Maxwell's equations determine whether such a particle resonance can build up. These resonances are often referred to as the surface modes of the particle [61].

The plasmon resonances are analytically known only for simple geometries, such as a sphere or a cylinder [58]. In a very small sphere, for instance, one single resonance can be excited, when $\varepsilon=-2(\mathrm{Ag}: \lambda \simeq 355 \mathrm{~nm}$, $\mathrm{Au}: \lambda \simeq 490 \mathrm{~nm})$, whereas a cylinder is in resonance when $\varepsilon=-1$ (Ag: $\lambda \simeq 337 \mathrm{~nm}, \mathrm{Au}: \lambda \simeq 253 \mathrm{~nm}$ ). With increasing particle size, these resonances are red-shifted and broadened, and additional higher-order resonances can be excited [58].

More than one single resonance can be excited in a non-regular structure, irrespective of its size. A cylinder with elliptical cross-section, for instance, exhibits two resonances, corresponding to the illumination directions along and normal to its major axis (Fig. 2a). Recently we demonstrated that triangular nanoparticles have several resonances for each illumination direction [50].

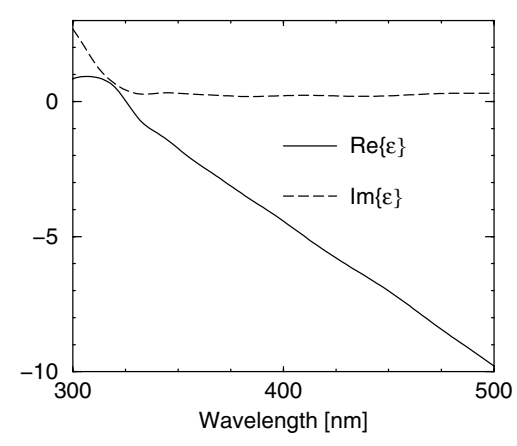

Fig. 1. Dispersion relation for silver used in our calculations. The real $\varepsilon^{\prime}$ and the imaginary $\varepsilon^{\prime \prime}$ parts of the permittivity are interpolated from the experimental data of Johnson and Christy [60] 

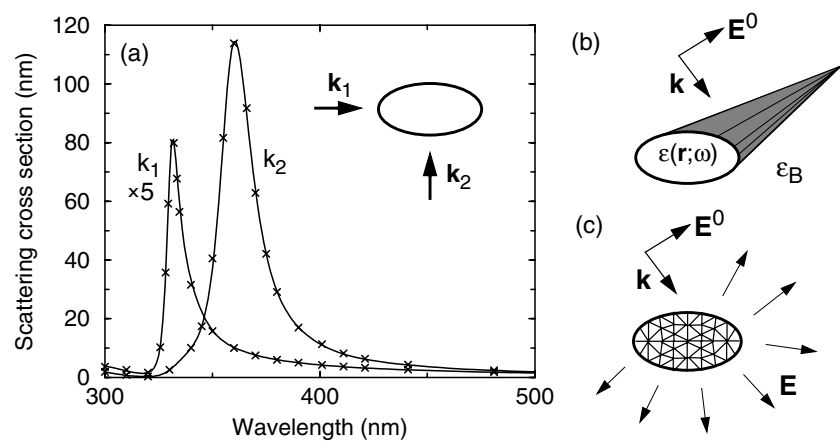

Fig. 2. (a) Scattering cross-section for an elliptical silver cylinder $(40 \mathrm{~nm} \times 20 \mathrm{~nm}$ section) computed with finite elements (solid line) and Mie theory (cross). Two orthogonal propagation directions $\boldsymbol{k}_{1}$ and $\boldsymbol{k}_{2}$ for the incident field, corresponding to two different resonances, are considered. The simulation of (b), an infinitely long nanowire, can be limited to its cross-section, (c). In our computational approach, the cross-section is discretized using triangular finite elements

Whether these different modes of a nanoparticle of arbitrary shape can be resolved, strongly depends on the material absorption (the imaginary part of $\varepsilon$ ): large absorption broadens the resonances, and can result in a nearly featureless band. Silver, compared with other metals that have their plasma frequency in the optical range, has a comparatively low absorption, and thus narrower resonances.

One may wonder whether the classical description of the material that we use, based solely on Maxwell's theory and a local dispersion relation, is appropriate for the small structures investigated here. Actually, it has been experimentally shown that this macroscopic approach is adequate for particle dimensions as small as $5 \mathrm{~nm}[37,59,62,63,64]$. Quantum effects must only be taken into account for smaller particles, using for example a jellium or quantum-chemical model $[59,65,66,67]$. For particles in the $5-20 \mathrm{~nm}$ range, the dispersion relation depends noticeably on the particle geometry, since the electron mean free path decreases as electron scattering at the surface becomes more important $[37,62,63,64]$. However, for the silver particles investigated here, only the imaginary part of the permittivity increases slightly, and the bulk values of permittivity still represent a good approximation. This point will be addressed in greater detail in Sect. 6 .

\section{$2.2 \quad$ Scattering Problem}

As established in the previous section, Maxwell's equations are well suited to the study of the plasmon resonances of silver particles in the $20-50 \mathrm{~nm}$ range, and we shall use the experimentally obtained permittivity values from Johnson and Christy [60]. For non-magnetic media, Maxwell's equations reduce 
in the frequency domain to the vectorial wave equation, which is formally solved by the volume integral equation:

$$
\boldsymbol{E}(\boldsymbol{r} ; \omega)=\boldsymbol{E}^{0}(\boldsymbol{r} ; \omega)+\int_{V} \mathrm{~d} \boldsymbol{r}^{\prime} \boldsymbol{G}^{\mathrm{B}}\left(\boldsymbol{r}, \boldsymbol{r}^{\prime} ; \omega\right) \cdot k_{0}^{2}\left[\varepsilon\left(\boldsymbol{r}^{\prime} ; \omega\right)-\varepsilon_{\mathrm{B}}\right] \boldsymbol{E}\left(\boldsymbol{r}^{\prime} ; \omega\right) .
$$

Here, $\boldsymbol{E}^{0}(\boldsymbol{r} ; \omega)$ is the incident electric field with vacuum wavenumber $k_{0}=$ $\omega / c, \boldsymbol{E}(\boldsymbol{r} ; \omega)$ is the unknown total scattered field, $\varepsilon(\boldsymbol{r} ; \omega)$ is the particle permittivity and $\varepsilon_{\mathrm{B}}$ is that of the background. The dyadic $\boldsymbol{G}^{\mathrm{B}}\left(\boldsymbol{r}, \boldsymbol{r}^{\prime} ; \omega\right)$ is the Green's tensor associated with the infinite homogeneous background [68]. This formalism can also be used when the background is a surface or a stratified medium $[69,70]$. Integrating the scattered field over all scattering angles provides the scattering cross-section (SCS) associated with the particle [58]. At the plasmon resonance the SCS shows distinct peaks, as the amount of scattered light increases abruptly. By repeating this calculation for different illumination frequencies $\omega$ with the corresponding permittivities $\varepsilon(\boldsymbol{r} ; \omega)$ one obtains the spectral response of the particle, as illustrated in Fig. 2a.

This so-called frequency-domain approach allows us, therefore, to take into account an arbitrary dispersion relation for the particle. The drawback is that the calculations must be repeated for each frequency where the particle response is required. An alternative approach, which provides the response of the particle over a certain frequency range in a single calculation, consists in using a time-domain technique such as the finite-difference time-domain technique [71]. In this case the dispersion relation must be included directly in the algorithm, which is only possible for the most simplistic dispersion models.

For infinitely long particles (cylinder, nanowire) under arbitrary illumination $\boldsymbol{E}^{0}$ (Fig. 2b), the scattering problem can be reduced to one section of the particle (Fig. 2c), [68]). We shall only consider illumination where the particles are illuminated in the plane of the figures with the electric field in the same plane (transverse-electric (TE) polarization). For transverse-magnetic (TM) polarization, where the incident magnetic field is in the plane, plasmons cannot be excited.

Equation (1) forms the basis for several different computational techniques for scattering calculations. The most widely used technique, the coupled dipole approximation (CDA), also known as the discrete dipole approximation (DDA), corresponds to solving (1) with finite differences $[46,47,48,68]$ $[72,73,74]$. Unfortunately, experience shows that for high-permittivity scatterers, or small scatterers (less than $100 \mathrm{~nm}$ size) that support plasmon resonances, this technique does not converge properly $[10,47,75]$. This means that increasing the number of discretization elements does not always improve the accuracy of the results. Worse, there can be cases where a thin mesh produces less results of the accurate than a coarse one [75]. This implies that subtle features of the resonance spectrum cannot be resolved and it is not possible to obtain a quantitative description of the field distribution in the close 
vicinity of the particle. However, the CDA/DDA can provide useful insight into the plasmon resonances for larger particles.

Recently, we became convinced that the flaw of the CDA/DDA is related to the singular behavior of the Green's tensor in (1) for $\boldsymbol{r} \rightarrow \boldsymbol{r}^{\prime}$. It is difficult to handle this singularity properly using a finite differences discretization because it assumes a constant field over each mesh. Refining the mesh requires the computation of the Green's tensor for neighboring points $\boldsymbol{r}$ and $\boldsymbol{r}^{\prime}$ that become closer and closer, which leads to exploding values for $\boldsymbol{G}^{\mathrm{B}}\left(\boldsymbol{r}, \boldsymbol{r}^{\prime} ; \omega\right)$. To overcome this difficulty, we developed a new technique based on finite elements [49]. The arbitrary particle section is discretized using triangles, and the unknown field $\boldsymbol{E}(\boldsymbol{r} ; \omega)$ is expanded into a sum of basis functions defined on each triangle (Fig. 2c). A Galerkin test procedure is then used to obtain a system of algebraic equations for the unknown field. We refer the reader to [49], where this technique is described in detail. Let us only mention that the use of finite elements allows us to handle exactly the singularity of the Green's tensor using generalized functions.

Since the spectrum of resonances for non-regularly shaped particles is not known, it is very important to first assess the suitability of this technique for investigating plasmon resonances. To do so, we resort to geometries where a reference solution exists. Figure 2a illustrates the accuracy of this approach for an elliptical particle. The entire spectrum agrees perfectly with that obtained semi-analytically using Mie's theory [58] and the location, magnitude and width of both plasmon resonances are reproduced. To quantify the accuracy of this technique, we show in Fig. 3 the error on the SCS as a function of the number of discretization elements. This error is defined as the square of the norm of the difference between the scattered field obtained with Mie's theory and that obtained with finite elements. Accurate results are already obtained with few discretization elements and, contrary to the CDA/DDA, it is always possible to increase the results accuracy by refining the mesh (Fig. 3). A key component in achieving these highly accurate results is the new regularization scheme that was developed to extract the singularity of the

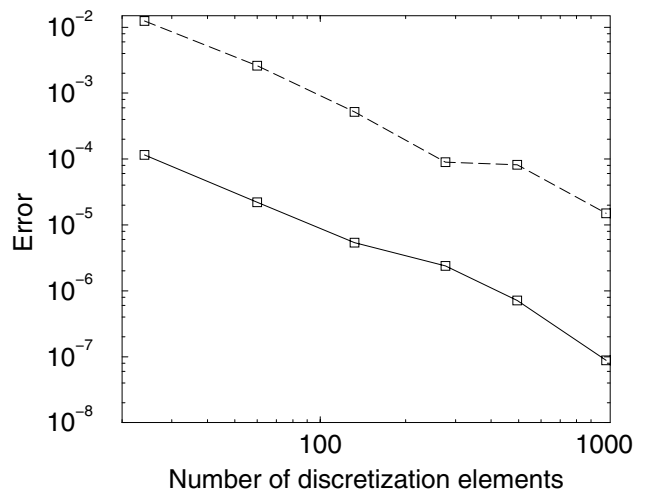

Fig. 3. Convergence of our finiteelement technique. The absolute error as a function of the number of discretization elements is reported. Two cylinders with permittivity $\varepsilon=16.64+0.23 i$ (silicon, dashed line) or $\varepsilon=4.0$ (solid line) are used as test objects. Their diameter is $100 \mathrm{~nm}$ and the incident wavelength $\lambda=546 \mathrm{~nm}$; TE polarization 
Green's tensor. This regularization scheme was also extended to neighboring elements, which further improves the overall accuracy and convergence [49].

The non-regular cross-sections investigated in this chapter were discretized with two to three thousand triangular elements. We verified that this discretization number was large enough so that the near-field distributions did not depend on it. We will consider nanowires with a polygonal section; dealing with sharp corners introduces additional numerical difficulties, since the field becomes singular at short distances from an infinitely sharp, perfectly conducting corner [76]. However, the sharpness of a real particle is limited by surface and boundary energies; therefore, we have rounded off each corner by $0.25 \mathrm{~nm}$, providing a more realistic model and removing the numerical instabilities. It is demonstrated in Sect. III. D of [52] that this minute corner smoothing does not at all influence the plasmon resonance SCS or near-field distribution.

\section{Relation Between Shape and Resonance Spectrum}

Having assessed in the previous section the suitability of our approach for the simulation of plasmon resonances in metallic nanowires, we consider in this section wires with a non-regular shape and investigate the relationship between that shape and the spectrum of resonances supported by the particle.

Figure 4 shows the scattering cross-section (SCS) from nanowires having cross-sections corresponding to that of a circle, a hexagon, a pentagon, a square and a triangle. The illumination direction is along one of the particle's symmetry axes. Two different sets of simplexes are considered: in Fig. 4a all particles have the same area as a $20 \mathrm{~nm}$ circle, whereas in Fig. 4b they have that of a $50 \mathrm{~nm}$ circle. Since all particles in the same figure part have the same area, their SCS should be comparable.
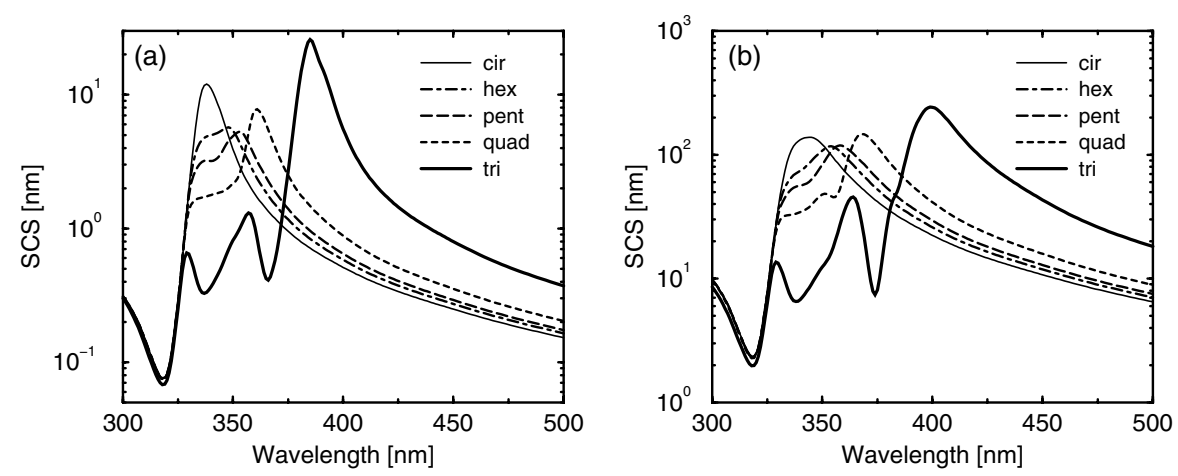

Fig. 4. SCS for (a) $20 \mathrm{~nm}$ and (b) $50 \mathrm{~nm}$ simplexes of varying symmetry (circle, hexagon, pentagon, square and triangle) 
Let us first consider the $20 \mathrm{~nm}$ simplexes (Fig. 4a). For the circle we recover the well-known result of a single resonance, at $\lambda=338 \mathrm{~nm}$ (corresponding to $\varepsilon=-1.07+0.29 i)$. The full-width half-maximum (FWHM) of the resonance is about $25 \mathrm{~nm}$. As can be seen in Fig. 4a, the structure of the SCS becomes more complex for less regular particles. The main resonance is red-shifted from $\lambda=338 \mathrm{~nm}$ (circle) to $\lambda=350 \mathrm{~nm}$ (hexagon), $\lambda=357 \mathrm{~nm}$ (pentagon), $\lambda=361 \mathrm{~nm}$ (square) and $\lambda=385 \mathrm{~nm}$ (triangle). An additional resonance appears for all the non-circular particles. Moreover, a third resonance can be observed for the triangle at $\lambda=357 \mathrm{~nm}$. The influence of the illumination direction on the SCS is very small, due to the symmetry of the simplexes (not shown).

The large SCSs that occur at the plasmon resonances are associated with large field amplitudes at the vicinity of the particle. As for the SCS, the complexity of the field distribution increases when the symmetry of the particle decreases. For the circular particle, the amplitude is homogeneous inside the particle, about seven times the incident field, and rapidly decreases outside the particle. For the non-regular structures the field becomes strongly heterogeneous and the enhancement factor increases dramatically. At the main resonance, the field amplitude takes large values at the corners transverse to the incident wave vector. There the relative field amplitude for the $20 \mathrm{~nm}$ simplexes (Fig. 4a) exceeds 20 for the hexagon and the pentagon, 70 for the square and 150 for the triangle.

Let us now consider the larger $50 \mathrm{~nm}$ simplexes (Fig. 4b). Two main differences are observed with respect to the SCS of the smaller simplexes (Fig. 4a). First, for all shapes the main resonance is red-shifted (e.g. for the triangle it is now at $\lambda=399 \mathrm{~nm}$, compared to $\lambda=385 \mathrm{~nm}$ for the $20 \mathrm{~nm}$ triangle). Second, the resonance FWHM is roughly doubled (i.e. for the circle, it is now more than $50 \mathrm{~nm}$, compared to $25 \mathrm{~nm}$ previously). We also see in Fig. $4 \mathrm{~b}$ that an additional resonance can be resolved for the square at $\lambda=351 \mathrm{~nm}$, whereas two additional resonances start to emerge for the triangle at approximately $\lambda=350 \mathrm{~nm}$ and $\lambda=382 \mathrm{~nm}$.

The triangular particle appears to have a rather complex spectrum of plasmon resonances, much more complex than a square particle. To further investigate this effect, we report in Fig. 5 the SCS for nanowires with sections that evolve from a rectangular shape into a triangular one. The triangular section is such that it has the same area as a cylinder with a $20 \mathrm{~nm}$ diameter (Fig. 4a); the base is approximatively $17 \mathrm{~nm}$ and the height $34 \mathrm{~nm}$. The rectangle has the same dimensions and the intermediate particles have a short side with length $0.75,0.5$, respectively, 0.25 times that of the triangle height. Let us note that the resonance spectrum covers a broader wavelength range for the right-angled triangle (Fig. 5) than for a similar equilateral triangle (Fig. 4a). The magnitude of the resonances is also larger in the case of the lowest-symmetry particle. 


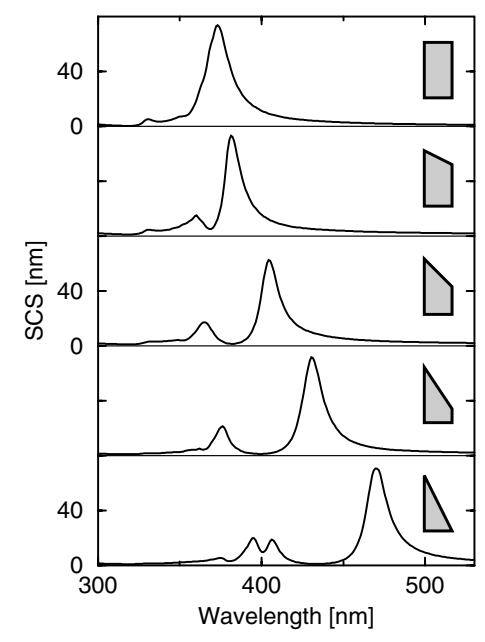

Fig. 5. SCS for nanowires with a cross-section evolving from a rectangular shape (top) to a triangular shape (bottom). The corresponding shape is shown in the inset; see the text for the dimensions. The illumination direction is along the lower left corner. The same vertical scale is used for the five curves

The FWHM for the main resonances in Fig. 5 is in the order of $15 \mathrm{~nm}$ and, surprisingly, does not vary much with the particle shape. It is, however, difficult to correlate the FWHM with the particle geometry only, as the main resonance occurs at a different wavelength, i.e. for a different permittivity (see Fig. 1). For example, the particle with the least-cut corner (Fig. 5, second from the top) has the narrowest resonance, FWHM=14 nm, but this resonance takes place at the wavelength where the absorption is minimum $\left(\varepsilon^{\prime \prime}=0.18\right)$, which narrows the resonance.

\section{Polarization Charge Distributions}

Corresponding to the complexity observed in the far field for the triangular particle, there is a very complicated near-field distribution which varies extremely rapidly when the illumination wavelength changes, as illustrated in the movies presented in [54].

To investigate these near-field effects in further details, let us consider a $10 \mathrm{~nm}$ base, $20 \mathrm{~nm}$ perpendicular right-angled triangular particle, whose SCS is shown in Fig. 6. The field distributions corresponding to the two resonances labeled in Fig. 6 are shown in Fig. 7a,b. In these figures, the field amplitude is color coded (unit incident amplitude), whereas the arrows give the direction of the electric field at a given time (the field is harmonic and oscillates over one period; movies illustrating this behavior are presented in $[55])$.

First, notice the dramatic field enhancement at close vicinity to the corner of the particle at the main resonance (Fig. 7a): the field reaches 200 times the amplitude of the incident field. This corresponds to an intensity enhancement of 40000 , or a Raman enhancement in excess of $10^{9}$ for a molecule immersed 


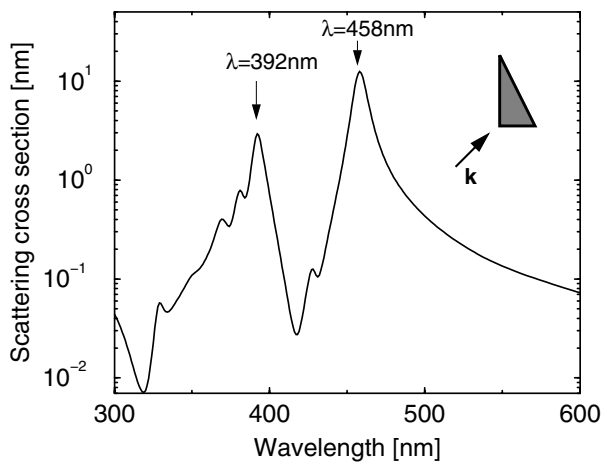

Fig. 6. SCS for a $10 \mathrm{~nm}$ base, $20 \mathrm{~nm}$ perpendicular right-angled triangle, as a function of the illumination wavelength

in this field distribution. This last point will be discussed in greater detail in Sect. 5 .

The field distributions observed in Fig. 7a,b are typical of the different types of behavior observed in non-regular particles. For the main resonance $(\lambda=458 \mathrm{~nm})$ the field radiates radially from the "hot" particle's corner, where the field is extremely homogeneous. For the next resonance $(\lambda=392 \mathrm{~nm})$, although the enhancement is comparable to the main resonance, the topology of the field is very different. Now the field seems to turn around the particle's top corner; its spatial variation is very rapid with an amplitude going from over 100 to 0 within a few nanometers inside the particle tip. This extremely fast variation of the field distribution actually illustrates the difficulties related to the simulation of plasmon resonances in nanoparticles with an arbitrary shape: It requires a technique which is stable and accurate enough to handle these important field gradients.

To understand better the intrinsic properties of the different resonances illustrated in Fig. 7a,b, we show in Fig. 7c,d the corresponding polarization charge distribution, which is given simply by the divergence of the electric field [77]. Of course, the particle does not become charged by the effect of the external field: a similar amount of positive and negative charge is induced on its surface, so that the particle always remains neutral. Over one period, these charges move around the particle, as illustrated in the movies presented in [55]. The polarization charge distributions shown in Fig. 7c,d correspond to snapshots at an arbitrary moment in time. Half a period later, the reverse charge distribution is observed (with plus charges where there were negative ones, and vice-versa).

As for the field distribution, we notice in Fig. 7c,d that each resonance is associated with a different charge distribution. In the main resonance, for $\lambda=458 \mathrm{~nm}$, charges of a given sign build up at the sharp corner, while opposite charges are distributed on the entire circumference of the particle (Fig. 7c). This distribution oscillates over time, the sign of the accumulated charges on the sharp corner changing every half-period. This explains why the corresponding field distribution is radial, with the electric field either 

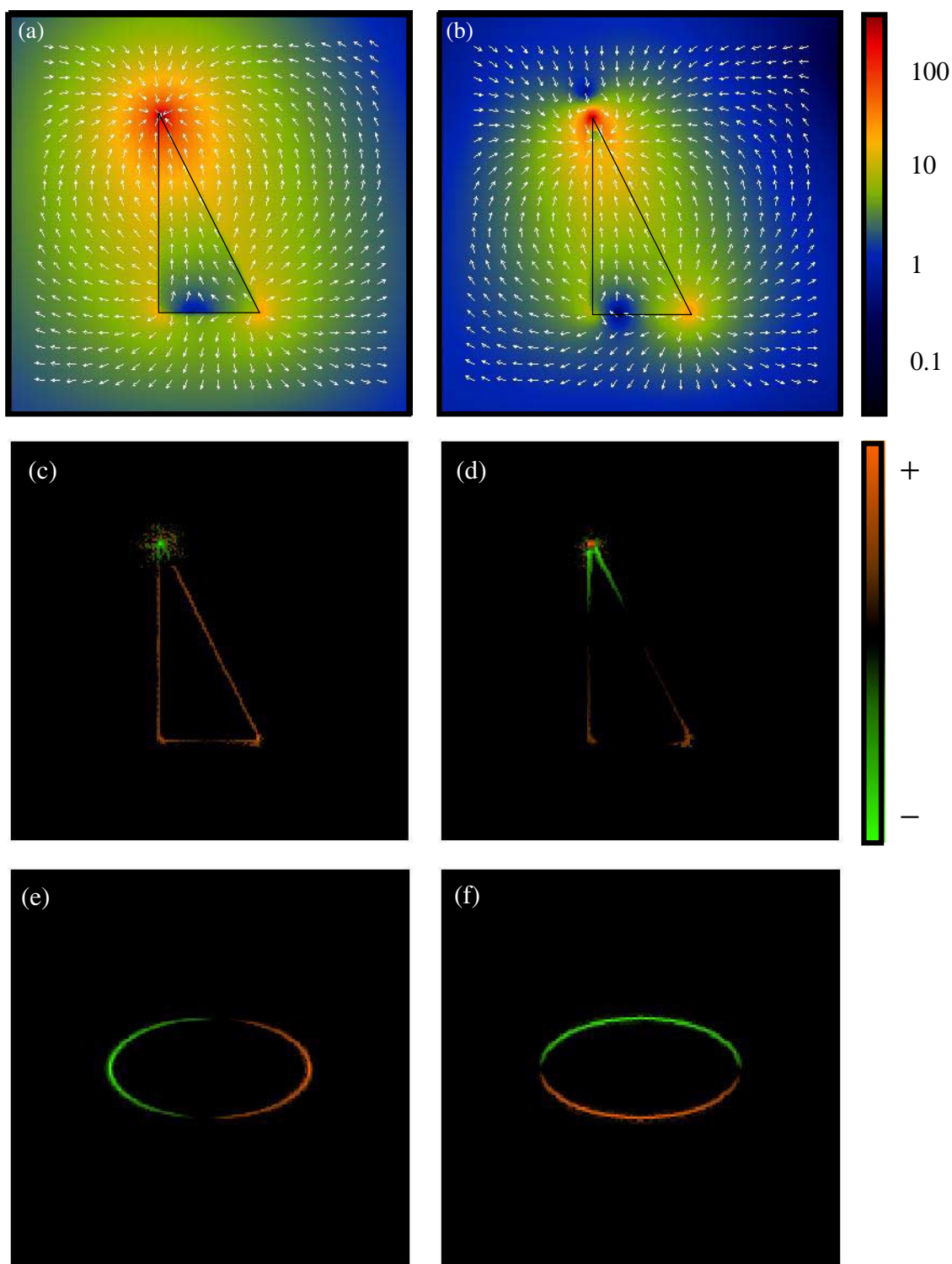

Fig. 7. (a),(b) Electric field amplitude distributions for the main $[(\mathbf{a}), \lambda=458 \mathrm{~nm}]$ and next-order $[(\mathbf{b}), \lambda=392 \mathrm{~nm}]$ resonances for a $10 \mathrm{~nm} \times 20 \mathrm{~nm}$ right-angled triangle (SCS in Fig. 6). The arrows show the orientation of the electric field. (c),(d) Polarization charge distributions associated with (a) and (b). (e),(f) Polarization charge distributions associated with the main $[(\mathbf{e}) \lambda=358 \mathrm{~nm}]$ and higher-order $[(\mathbf{f}) \lambda=331 \mathrm{~nm}]$ resonances for a $20 \mathrm{~nm} \times 10 \mathrm{~nm}$ elliptical particle 
pointing towards or away from this corner, which is characteristic of a point source.

For the next resonance, $\lambda=392 \mathrm{~nm}$, both charge species accumulate simultaneously at the sharp corner: one species accumulates at the very tip, while the species of opposite sign is distributed along the adjacent sides (Fig. 7d). This dipolar-like charge distribution determines the field at the sharp corner, where the field appears to turn around the corner as it does at the vicinity of a dipole (Fig. 7b).

For higher-order modes, a more complex charge distribution is observed, as described in [55]. This charge distribution is extremely useful for understanding the topology of the electric field, both for individual and interacting nanoparticles. For example, when two particles interact together, new plasmon resonances can appear. The topology of the corresponding field can be easily related to the charge distribution on both particles $[57,56]$.

To illustrate the topology of the charge distribution in a somewhat more intuitive case, Fig. 7e,f show the distributions associated with the two modes of an elliptical particle (SCS similar to that in Fig. 2a, although the particle is now smaller $)$. For the fundamental mode $(\lambda=358 \mathrm{~nm}$, Fig. 7e) the electric field is parallel to the major axis, corresponding to polarization charges accumulating at both ends of this axis. For the higher-order mode $(\lambda=331 \mathrm{~nm}$, Fig. 7f), the polarization charges are split vertically, following the polarization direction of the incident field.

The polarization charge distributions are also helpful for understanding the spatial extension of the electric field away from the particle surface. In Fig. 7, for example, one notices that the spatial extension of the field is larger for the fundamental mode (Fig. 7a) than for the next-order mode, where the field decreases much faster (Fig. 7b). This is not surprising, since the former mode is related to a point-like charge distribution (3D spatial variation $\sim 1 / r)$, whereas the latter is associated with a dipolar charge distribution $\left.\sim 1 / r^{3}\right)$. This difference of distance dependence as a function of excited resonance, i.e. as a function of illumination wavelength, could be demonstrated by the approach curves in scanning near-field optical microscopy (SNOM) experiments [51].

In that context of local probe microscopy, let us emphasize that the different plasmon resonances supported by a non-regular shape nanoparticle provide many different electromagnetic environments, while keeping the physical system unchanged. This could be used to suppress experimental artifacts by performing a series of measurements under different illumination wavelength and tracking the pure optical contrast [78].

Let us stress here that the large fields observed in Fig. 7a,b near the particle's corners are not produced by the lightning rod or tip effect [76]. The latter provides only a field amplification factor in the order of 5 to 10, even for very large permittivity values, as illustrated in Fig. 4c of [54]. 


\section{$5 \quad$ Field Enhancement and SERS}

In this section we discuss the near-field distributions associated with the plasmon resonances of the different simplexes, with emphasis on the local variation of the field amplitude around the particles. This variation plays a key role in the practical implementation of plasmon-resonant particles in different areas of nanoscience. For SERS in particular, the electromagnetic enhancement effectively experienced by a molecule depends on the location where the molecule is adsorbed on the metal, as well as the relative position of the Raman active site within the molecule. This becomes very important when the molecule is large and placed in a strongly inhomogeneous field. This was recently verified experimentally by van Duyne et al., who inserted different numbers of non-active linker molecules between the adsorption site of a molecule and the Raman active site [79]. In this experiment, the Raman signal was highly dependent on the number of linker molecules, and therefore on the distance between the surface and the Raman active site, particularly for non-regular (tetrahedral) nanoparticles. Further, when a large number of molecules are adsorbed on the same nanoparticle, a spectral shift in the particle resonances can be observed [80].

Figure 8 shows the maximum enhancement obtained at a distance of $1 \mathrm{~nm}$ from the surface as a function of the wavelength. The amplitude enhancement is shown on the left-hand axis and the corresponding Raman enhancement on the right-hand; both $20 \mathrm{~nm}$ and $50 \mathrm{~nm}$ simplexes are investigated. For molecules excited far from an electronic absorption band, the intensity of the Raman scattered light is proportional to the fourth power of the local electric field amplitude where the molecule is immersed [7]. Plasmon-resonant particles provide a convenient method of enhancing the electromagnetic fields, and therefore are ideal SERS substrates. A second enhancement mechanism, the so-called chemical enhancement, related to the adsorption of the molecule on
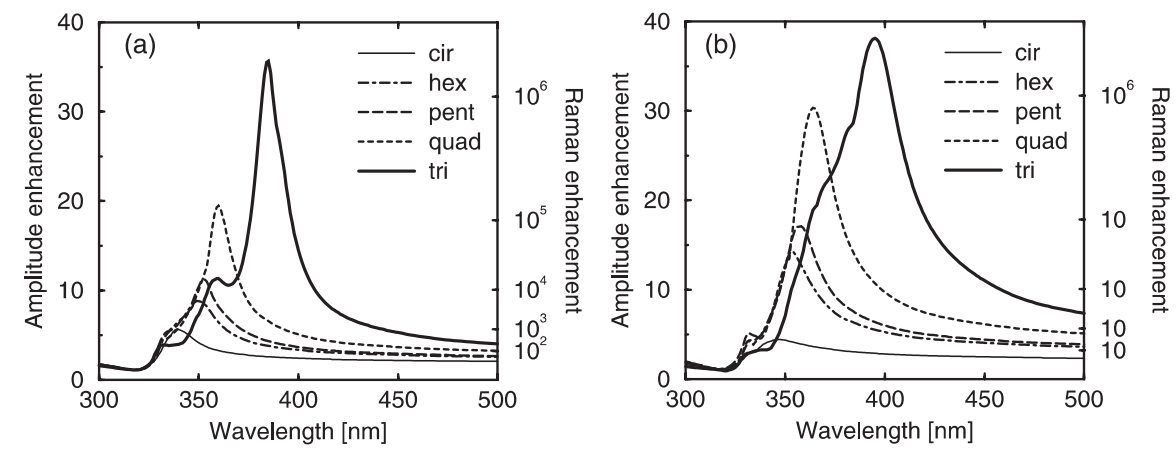

Fig. 8. Maximum amplitude enhancement for the (a) $20 \mathrm{~nm}$ and (b) $50 \mathrm{~nm}$ simplexes (SCS in Fig. 4). The right-hand scale shows the corresponding maximum Raman enhancement 
the metal, can also contribute to SERS $[11,12,81]$. However, electromagnetic enhancement is believed to make the major contribution to SERS.

We observe that the field enhancement, which is a near-field quantity, is strongly correlated to the SCS, a far-field quantity (compare Figs. 8 and 4). The position of the main resonance is the same, but the resonance width is broader in the enhancement diagram (Fig. 8) than in the SCS (Fig. 4). This is simply because in Fig. 8 we show the maximum amplitude enhancement around the particle, whereas in Fig. 4 we show the SCS, which is related to the field amplitude squared. The maximum amplitude enhancement increases considerably for more complex shapes: whereas it is 6 for the circle, it is about 35 for the triangle.

The differences between the simplex shapes is much more pronounced for the enhancement than for the SCS. For the $20 \mathrm{~nm}$ triangle the maximum Raman enhancement exceeds $10^{6}$, and for the square it is still about $10^{5}$, whereas it is below $10^{4}$ for the hexagon, the pentagon and the circle (Fig. 8a).

The field enhancement for the larger simplexes is shown in Fig. 8b, again at a distance of $1 \mathrm{~nm}$ from the surface. We observe that the correlation with the SCS diagram is now weaker. The maximum enhancement is comparable to that obtained previously for the smaller particles.

As discussed previously, the local variations of the near-field distribution determine the effective enhancement experienced by a molecule. This is investigated in Fig. 9, where we present the amplitude distribution as a function of the distance from the tip surface for the triangle, square and circle. The data correspond to the main resonance, and two particle sizes are considered.

For the circle, the field amplitude on the surface reaches 6.9 (4.5), for the small (large) circle. At $2 \mathrm{~nm}$ from the surface, the amplitude drops to 2.2 (1.7). For the triangle we observe a huge enhancement for both particle sizes: near the surface more than 100 in terms of amplitude ( $10^{8}$ Raman), and still more than $50\left(6 \times 10^{6}\right)$ at a half-nanometer distance (Fig. 9). For the square we obtain a similar behavior, although the amplitudes are about a factor of two smaller (the Raman enhancement being 16 times weaker). The strong

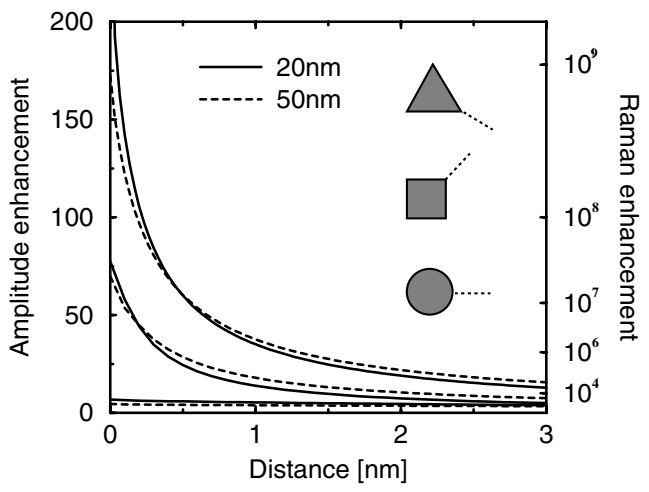

Fig. 9. Enhancement as a function of the distance from the corner (along the dashed line in the inset), for the main resonance of the $20 \mathrm{~nm}$ and $50 \mathrm{~nm}$ simplexes. The curves follow the particles order in the inset 
field-amplitude variations for such non-regular structures might explain the "hot spots" observed both in SERS experiments and in direct measurements of the locally enhanced field $[9,82]$.

Figure 9 indicates similar results for the maximum amplitude distribution around the $20 \mathrm{~nm}$ and the $50 \mathrm{~nm}$ simplexes. Although not shown here, this enhancement rapidly decreases for particle sizes above $50 \mathrm{~nm}$ [50]. For example, the maximum amplitude enhancement at the corner of a $100 \mathrm{~nm}$ triangle is only half that of the 20 or $50 \mathrm{~nm}$ particles [54].

Within our model we therefore observe that for a given particle shape the maximum field enhancement increases with decreasing particle sizes down to $50 \mathrm{~nm}$ and then remains fairly constant. Note that this maximum enhancement occurs at different wavelengths for different sizes.

Although the local maximum enhancement is similar for particles in the 20-50 nm range, the average over the entire particle may differ with the particle size. This is important for SERS experiments in which large numbers of molecules are used, since the measured Raman signal is proportional to the average Raman enhancement on the surface. This is illustrated in Fig. 5 of [54], where the overall near-field distribution for particles between 10 and $100 \mathrm{~nm}$ is shown. We shall now discuss this average enhancement in further details.

In Fig. 10 we show the average enhancement for the $20 \mathrm{~nm}$ and $50 \mathrm{~nm}$ simplexes. This average value is obtained by taking between 500 and 800 points (depending on the particle shape) distributed regularly around the particle, at a distance of $1 \mathrm{~nm}$ from the surface. Note that the average amplitude (Fig. 10a,b) and the average Raman enhancement (Fig. 10c,d) must now be represented on separate graphs.

In Fig. 10a we observe that the average field amplitude is strongly correlated with the SCS (Fig. 4a), both with respect to the wavelength and the width of the different resonances. The average field amplitude enhancement is quite similar for the circle, the hexagon and the pentagon: about 5.5 times the initial amplitude. For the square it is about 7.5 at the corresponding main resonance wavelength, whereas it reaches almost 12 for the triangle.

The average Raman enhancement for the $20 \mathrm{~nm}$ simplexes, as shown in Fig. 10c, is less than $10^{3}$ for the circle, becomes larger for the hexagon and the pentagon, and reaches about $10^{4}$ for the square and almost $10^{6}$ for the triangle (always at the corresponding main resonance wavelength). It is important to realize that, due to the rapid variations of the field, the maximum field amplitude on the particle circumference dominates the average Raman enhancement, as the fourth power of the field is taken. This is the reason why the average Raman enhancement is larger than the fourth power of the corresponding average field enhancement (Fig. 10a,c).

Similar results for the $50 \mathrm{~nm}$ simplexes are shown in Fig. 10b,d. Comparing Fig. 10a,b, we observe that these larger simplexes produce a smaller average field enhancement than their $20 \mathrm{~nm}$ counterparts. This is particularly the 

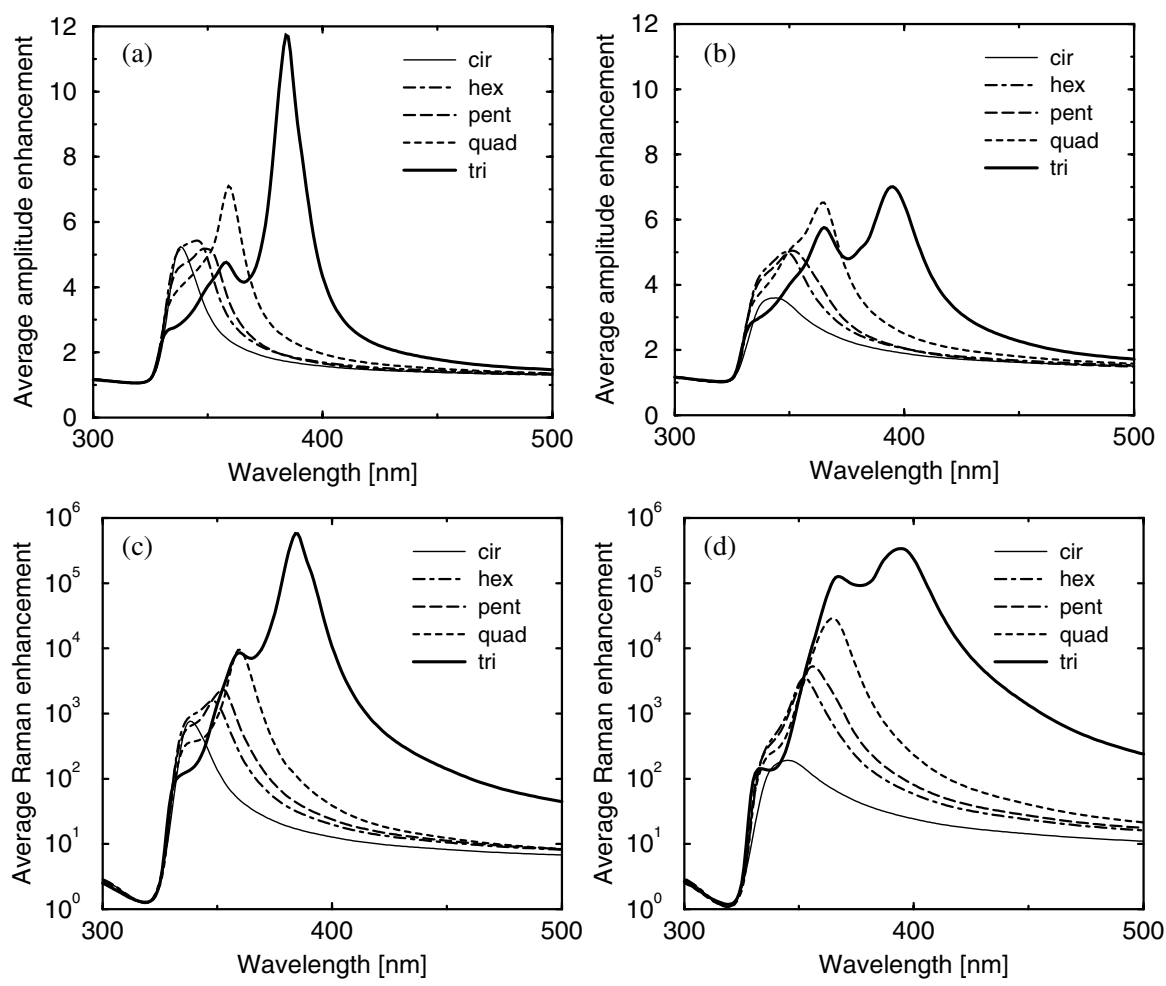

Fig. 10. (a),(b) Average amplitude enhancement and (c),(d) average Raman enhancement, computed at a $1 \mathrm{~nm}$ distance from the surface of the $20 \mathrm{~nm}$ simplexes (a),(c) and the $50 \mathrm{~nm}$ simplexes (b),(d)

case for the triangular nanowires, where the maximum average enhancement drops from almost 12 to less than 7 times the illumination amplitude. (For the other shapes, the decrease of the maximum average enhancement is less than $15 \%$.) For the average Raman enhancement we also observe a smaller enhancement for the $50 \mathrm{~nm}$ circular particle than for its $20 \mathrm{~nm}$ counterpart. For the hexagon, pentagon and square particles, the average Raman enhancement is somewhat larger for the $50 \mathrm{~nm}$ than for the $20 \mathrm{~nm}$ simplexes (Fig. 10c,d). For the triangular particle, the average Raman enhancement at the main resonance is slightly weaker for the $50 \mathrm{~nm}$ particle than for the $20 \mathrm{~nm}$ one, whereas for the next resonance it is more than ten times stronger. Again, this can be understood with the larger maximum Raman enhancement, as observed in Fig. 8. This larger maximum value outweighs the fact that, for the $50 \mathrm{~nm}$ particles, the field amplitude is on average smaller than for the $20 \mathrm{~nm}$ particles.

This illustrates the complexity of the interpretation of Raman experiments in which large numbers of molecules are used. The fact that the local 
Raman enhancement (Fig. 8) can be much stronger than the average enhancement (Fig. 10) indicates that in such an experiment a very limited number of molecules can contribute the major part of the SERS effect. Let us finally note that the distance between the active Raman site and the surface can also influence the respective magnitude of the local and average enhancements. As a matter of fact, Fig. 9 indicates that at very short distances from the surface, the field is stronger for the $20 \mathrm{~nm}$ simplexes. In that case, both the average amplitude and the average Raman enhancements are larger for the $20 \mathrm{~nm}$ simplexes.

The large number of resonances observed in non-regular particles opens interesting applications. For example, by tuning the wavelength of the illumination light, it is possible to modify the field distribution in the system and influence also the Raman enhancement, in particular its distance dependence, as illustrated in Fig. 11. The possibility to address different resonances by tuning the illumination wavelength can also prove useful for other specific applications, such as apertureless SNOM [83].

In Fig. 11 we show the field amplitude as a function of the distance from the corner for the three resonances of the $50 \mathrm{~nm}$ triangle (see Fig. 4b for the corresponding SCS). We observe a similar enhancement of about 150 close to the surface for the main resonance $(\lambda=399 \mathrm{~nm})$ and the resonance at $\lambda=364 \mathrm{~nm}$. However, the field decays more rapidly for the latter resonance (Fig. 11). As explained in Sect. 4, this behavior can be related to the polarization charge distribution associated with both resonances.

The extremely important dynamic range observed in Fig. 11 can be useful in an apertureless SNOM experiment, where the tip is vibrated vertically above the sample. It could provide an extremely good signal-to-noise ratio and facilitate the optical feedback of the tip motion.

The other deciding factor that determines the resolution in a SNOM experiment is the lateral field confinement. In [51] we showed that the field distribution obtained with a non-regular nanoparticle remains extremely well confined. At distances between $1 \mathrm{~nm}$ and $5 \mathrm{~nm}$ from the tip, the full width half-maximum (FWHM) of the lateral field distribution varies between $2 \mathrm{~nm}$

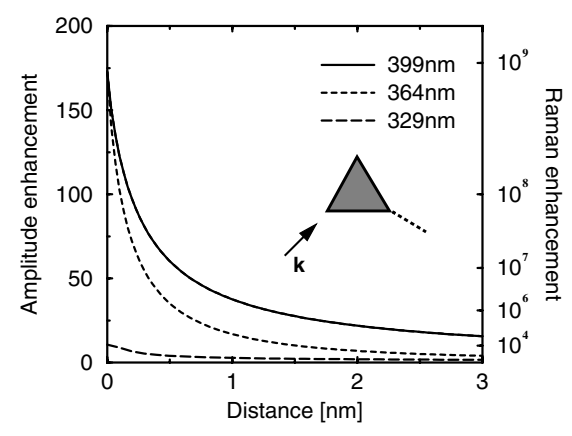

Fig. 11. Field amplitude enhancement as a function of the distance from the corner of the $50 \mathrm{~nm}$ triangle along the dashed line in the inset, for three different resonance wavelengths (SCS in Fig. 4b) 
and $10 \mathrm{~nm}$ [51]. In the close vicinity of the particle, the field is confined over dimensions much smaller than the particle size, as illustrated in Fig. 7a,b.

\section{Influence of the Model Permittivity and of the Background}

The previous results emphasize how complex the study of plasmon resonances can be. In particular, the comparison between different particle shapes is quite delicate, as the corresponding resonances occur at specific frequencies, with different permittivities. In particular, $\varepsilon^{\prime \prime}-$ which accounts for the absorption - strongly influences the resonance width and the near-field enhancement. To clarify this point, we concentrate in this section on the triangular particle investigated in Fig. 5 and study the influence of the absorption on the SCS and near-field enhancement.

The definition of the permittivity for a nanoscopic particle is not trivial. For particles smaller than $2 \mathrm{~nm}$, the classical description breaks down and jellium or quantum-chemistry models must be used $[65,67]$. For the particles in the 10-50 nm range considered here, a local permittivity can still be used to describe the metal, but the bulk value must be modified as the particle size becomes comparable to the bulk electron mean free path (the average path of a conduction electron between two scattering processes). In that case, electron scattering at the particle surface becomes a dominant effect. It reduces the electron mean free path, which in turn leads to an increase of the imaginary part of the permittivity. This has been shown experimentally for spherical particles by Kreibig et al. [59,63,84]. They showed that for particle sizes below $40 \mathrm{~nm}$ the resonance width becomes broader, and in particular they found that near the plasma resonance frequency the imaginary part can be well described by the relation:

$$
\varepsilon^{\prime \prime}=0.23+\frac{2.64}{a},
$$

where $a$ is the particle size in nanometers [58,84].

More recently, the electron dynamics in silver and copper particles in the 5-10 nm range was investigated using femtosecond laser pulses [64]. It was found that for a $6.5 \mathrm{~nm}$ particle size the resonance width is between 3 and 4 times larger than expected from the bulk permittivity, thereby indicating a larger absorption. The resonance was also slightly blue shifted, indicating that the real part of the permittivity can also be affected for such a small particle size [64]. Similar results have been obtained for silver particles in the $3-13 \mathrm{~nm}$ range by another group [85]. For spherical particles in the $20 \mathrm{~nm}$ range investigated here, only the imaginary part of the permittivity seems to be affected by the particle size [58,59].

No experimental data are available for the modification of the dispersion relation in non-spherical particles of a given shape. One can, however, expect 
that corners produce additional electron scattering, thereby locally increasing the absorption. Following (2), and to gain insights in the influence of the absorption on the particle response, we investigate three different absorptions.

Figure 12 shows the SCS and the near-field distribution for the same right-angled triangular particle as in Fig. 5, using three different values for the imaginary part $\varepsilon^{\prime \prime}$ of the permittivity. The original values of Johnson and Christy, as measured for the bulk, produce four well-resolved resonances. The main resonance is quite narrow $(\mathrm{FWHM}=16 \mathrm{~nm})$. This resonance broadens when the absorption increases: it is FWHM $=28 \mathrm{~nm}$ for $\varepsilon^{\prime}+i 2 \varepsilon^{\prime \prime}$ and FWHM $=52 \mathrm{~nm}$ for $\varepsilon^{\prime}+i 4 \varepsilon^{\prime \prime}$ (Fig. 12a).

Further, the absorption has a strong influence on the higher-order resonances, which progressively merge into one broad resonance, as the imaginary part of the permittivity increases. Note in Fig. 12a that the resonances are not shifted, since only the imaginary part of the permittivity changes (the plasmon resonance condition being solely determined by the real part of the permittivity).

The influence of the damping factor on the near-field enhancement is investigated in Fig. 12b, for the same particle. This effect is quite dramatic, as the field amplitude for the main resonance at $0.5 \mathrm{~nm}$ from the surface drops from $112\left(\varepsilon^{\prime}+i \varepsilon^{\prime \prime}\right)$ to $65\left(\varepsilon^{\prime}+i 2 \varepsilon^{\prime \prime}\right)$ and $36\left(\varepsilon^{\prime}+i 4 \varepsilon^{\prime \prime}\right)$. This influence of the particle absorption remains visible at larger distances from the surface (Fig. 12b). A similar behavior is observed for higher-order resonances, although in that case the field dies out rapidly when one moves away from the surface [53].

Let us finally study the influence of the particle background on the resonance spectrum. This is evidently important for experiments in solutions, as well as for many experiments on surfaces, as a thin water layer is often
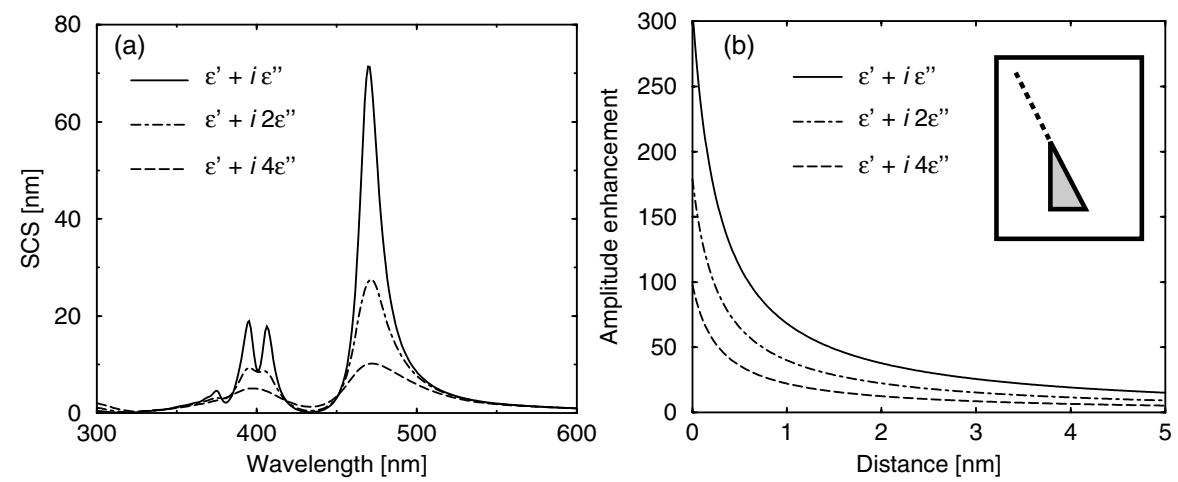

Fig. 12. (a) SCS for a triangular particle using different permittivities with increasing imaginary parts $\varepsilon^{\prime \prime}$ (absorption factor). (b) Field amplitude enhancement as a function of the distance from the corner (see inset), for the main resonance of the particle in $(\mathbf{a})(\lambda=470 \mathrm{~nm})$ 


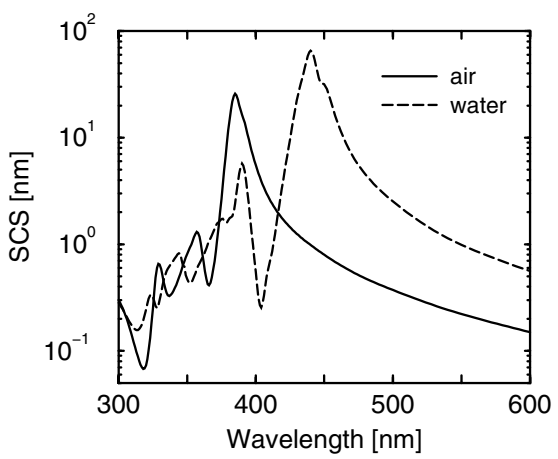

Fig. 13. SCS for a triangular particle (side $20 \mathrm{~nm}$ ) in air and in water backgrounds

present in that case. Figure 13 shows the SCS for a triangular particle in air $\left(\epsilon_{\mathrm{B}}=1\right)$ and in water $\left(\epsilon_{\mathrm{B}}=1.78\right.$, water dispersion is negligible in the visible range and the permittivity can be assumed constant [86]).

We observe that for a higher background permittivity the resonance spectrum is red-shifted and broadened, very similarly to the case where the particle size increases (e.g. compare with Fig. 4a,b). As a matter of fact, for larger background permittivity, the particle simply becomes larger with respect to the effective illumination wavelength in the background; retardation - which is responsible for the size effect - then comes into play for smaller particle sizes.

\section{Conclusions and Outlook}

Our results shed new light on the very old problem of the relation between a nanoparticle shape and its plasmon resonance spectrum. For the first time it is clearly demonstrated for particles with a non-regular shape, that the number of resonances strongly depends on the form of the particle. The higher the particle symmetry, the simpler is its spectrum (e.g. a small cylindrical particle exhibits only one resonance, whereas a square has two and an equilateral triangle at least three distinct resonances). Several additional resonances are observed for right-angled triangular particles with a high perpendicular-to-base ratio.

These complex scattering cross-sections are also associated with a dramatic near-field enhancement in the close vicinity of the particle. We found the strongest enhancement for particles with dimensions smaller than $50 \mathrm{~nm}$. Right-angled triangular particles produce a field amplitude many hundred times stronger than the incident field at short distances from the surface. This dramatic near-field enhancement corresponds to a huge Raman enhancement, similar to that required by recent SERS experiments, where single molecule detection was possible $[8,9,87]$.

The strong localization of the near-field at specific positions around the metallic particle, as well as its rapid decay when one moves further away 
from the particle surface, can also explain the "hot-spots" observed in SERS experiments, where specific sites appear to be particularly active. This is also confirmed by our results on the average Raman enhancement, which indicate that a limited number of molecules can contribute the major part of the SERS signal, even in experiments in which large numbers of molecules are used.

The topology of the field distribution inside and in the vicinity of the particle can be easily understood in terms of the polarization charges. To each plasmon resonance corresponds a specific charge distribution, which produces a particular field. The field distribution associated with each plasmon resonance is different, in particular its decay rate as one moves away from the particle surface. This can be useful for near-field optical microscopy and other local probe techniques that rely on strongly localized electromagnetic fields.

The nanowires investigated here can now be fabricated in a controlled manner using different nanofabrication techniques. A direct experimental verification of our results is therefore possible. The next challenge however, is a fully three-dimensional (3D) model, which can handle arbitrary shape particles. Our 2D results indicate that at the plasmon resonance the field distribution strength depends on the confinement of the polarization charges at specific locations in the particle. For nanowires, this confinement can take place in the two transverse dimensions. Therefore, for 3D particles, where the charges can be confined in all three directions, we expect an even stronger enhancement.

\section{Acknowledgements}

It is a pleasure to acknowledge J. P. Kottmann who developed the finite elements technique and calculated most of the configurations presented here in the course of his Ph.D. I am also indebted to D. R. Smith and S. Schultz for many stimulating discussions.

\section{References}

1. M. Kerker: Founding fathers of light scattering and surface-enhanced Raman scattering, Appl. Opt. 30, 4699 (1991) 183

2. R. Jin, Y.W. Cao, C. A. Mirkin, K. L. Kelly, G. C. Schatz, J. G. Zheng: Photoinduced conversion of silver nanospheres to nanoprism, Science 294, 1901 (2001) 184

3. J. J. Mock, M. Barbic, D. R. Smith, D. A. Schultz, S. Schultz: Shape effects in plasmon resonance of individual colloidal silver nanoparticles, J. Chem. Phys. 116, 6755 (2002) 184

4. M. Fleischmann, P. J. Hendra, A. J. McQuillan: Raman-spectra of pyridine adsorbed at a silver electrode, Chem. Phys. Lett. 26, 163 (1974) 184

5. D. L. Jeanmaire, R.P. van Duyne: Surface Raman spectroelectrochemistry. 1. Heterocyclic, aromatic, and aliphatic-amines adsorbed on anodized silver electrodes, J. Electroanal. Chem. 84, 1 (1977) 184 
6. H. Metiu: Surface enhanced spectroscopy, Prog. Surf. Sci. 17, 153 (1984) 184

7. M. Moskovits: Surface-enhanced spectroscopy, Rev. Mod. Phys. 57, 783 (1985) 184, 196

8. K. Kneipp, Y. Wang, H. Kneipp, L. T. Perelman, I. Itzkan, R. R. Dasari, M. S. Feld: Single molecule detection using surface-enhanced Raman scattering, Phys. Rev. Lett. 78, 1667 (1997) 184, 203

9. S. Nie, S.R. Emory: Probing single molecules and single nanoparticles by surface-enhanced Ramsn scattering, Science 275, 1102 (1997) 184, 198, 203

10. H. Xu, E. J. Bjerneld, M. Käll, L. Börjesson: Spectroscopy of single Hemoglobin molecules by surface enhanced Raman scattering, Phys. Rev. Lett. 83, 4357 (1999) 184, 188

11. A. Otto, I. Mrozek, H. Grabhorn, W. Akemann: Surface-enhanced Raman scattering, J. Phys. C 4, 1143 (1992) 184, 197

12. P. Kambhampati, C. M. Child, M. C. Foster, A. Campion: On the chemical mechanism of surface enhanced Raman scattering: Experiment and theory, J. Chem. Phys. 108, 5013 (1998) 184, 197

13. J. R. Krenn, A. Dereux, J. C. Weeber, E. Bourillot, Y. Lacroute, J. P. Goudonnet, G. Schider, W. Gotschy, A. Leitner, F. R. Aussenegg, C. Girard: Squeezing the optical near-field by plasmon coupling of metallic nanoparticles, Phys. Rev. Lett. 82, 2590 (1999) 184

14. J.-C. Weeber, A. Dereux, C. Girard, J.R. Krenn, J.-P. Goudonnet: Plasmon polaritons of metallic nanowires for controlling submicron propagation of light, Phys. Rev. B 60, 9061 (1999) 184

15. T. Yatsui, M. Kourogi, M. Ohtsu: Plasmon waveguide for optical far/near-field conversion, Appl. Phys. Lett. 79, 4583 (2001) 184

16. J. Tominaga, T. Nakano, N. Atoda: Super-resolution structure for optical data storage by near-field optics, Proc. SPIE 3467, 282 (1999) 184

17. L. Men, J. Tominaga, H. Fuji, Q. Chen, N. Atoda: High-density optical data storage using scattering-mode super-resolution near-field structure, Proc. SPIE 4085, $204(2001) \quad 184$

18. J. Tominaga, C. Mihalcea, D. Büchel, H. Fukuda, T. Nakano, N. Atoda, H. Fuji, T. Kikukawa: Local plasmon photonic transistor, Appl. Phys. Lett. 78, 2417 (2001) 184

19. K. Bromann, C. Félix, H. Brune, W. Harbich, R. Monot, J. Buttet, K. Kern: Controlled deposition of size-selected Silver nanoclusters, Science 274, 956 (1996) 185

20. D. M. Kolb, R. Ullmann, T. Will: Nanofabrication of small copper clusters on gold(111) electrodes by a scanning tunneling microscope, Science 275, 1097 (1997) 185

21. Y.-Y. Yu, S.-S. Chang, C.-L. Lee, C. R. C. Wang: Gold nanorods: Electrochemical synthesis and optical properties, J. Phys. Chem. B 101, 6661 (1997) 185

22. K. Abe, T. Hanada, Y. Yoshida, N. Tanigaki, H. Takiguchi, H. Nagasawa, M. Nakamoto, T. Yamaguchi, K. Yase: Two-dimensional array of silver nanoparticles, Thin Solid Films 327-329, 524 (1997) 185

23. D. Y. Petrovykh, F. J. Himpsel, T. Jung: Width distribution of nanowires grown by step decoration, Surf. Science 407, 189 (1998) 185

24. G. L. Che, B. B. Lakshmi, E. R. Fisher, C. R. Martin: Carbon nanotubule membranes for electrochemical energy storage and production, Nature 393, 346 (1998) 185 
25. J. Viereck, F. Stietz, M. Stuke, T. Wenzel, F. Träger: The role of surface defects in laser-induced thermal desorption from metal surfaces, Surf. Sci. 383, 749 (1997) 185

26. J. Bosbach: Laser-based method for fabricating monodispersive metallic nanoparticles, Appl. Phys. Lett. 74, 2605 (1999) 185

27. I. Utke, P. Hoffmann, B. Dwir, E. Kapon, P. Doppelt: Focused electron beam induced deposition of gold, J. Vac. Sci. Technol. B 18, 3168 (2000) 185

28. A.P. Li, F. Müller, U. Gösele: Polycrystalline and monocrystalline pore arrays with large interpore distance in anodic alumina, Electrochem. Solid-State Lett. 3, 131 (2000) 185

29. D.-S. Wang, H. Chew, M. Kerker: Enhanced Raman scattering at the surface of a spherical particle, Appl. Opt. 19, 2256 (1980) 185

30. M. Kerker, D.-S. Wang, H. Chew: Surface enhanced Raman scattering (SERS) by molecules adsorbed at spherical particles: errata, Appl. Opt. 19, 4159 (1980) 185

31. P. K. Aravind, A. Nitzan, H. Metiu: The interaction between electromagnetic resonances and its role in spectroscopic studies of molecules adsorbed on colloidal particles or metal spheres, Surf. Sci. 110, 189 (1981) 185

32. P. W. Barber, R. K. Chang, H. Massoudi: Electrodynamic calculations of the surface-enhanced electric intensities on large Ag spheroids, Phys. Rev. B 27, $7251(1983) 185$

33. M. Inoue, K. Ohtaka: Enhanced Raman scattering by two-dimensional array of polarizable spheres, J. Phys. Soc. Jpn. 52, 1457 (1983) 185

34. K. T. Carron, W. Fluhr, M. Meier, A. Wokaun, H. W. Lehmann: Resonances of two-dimensional particle gratings in surface-enhanced Raman scattering, J. Opt. Soc. Am. B 3, 430 (1986) 185

35. R. Rojas, F. Claro: Theory of surface enhanced Raman scattering in colloids, J. Chem. Phys. 98, 998 (1993) 185

36. A. I. Vanin: Surface-amplified Raman scattering of light by molecules adsorbed on groups of spherical particles, J. Appl. Spectrosc. 62, 32 (1995) 185

37. K.-P. Charlé, L. König, S. Nepijko, I. Rabin, W. Schulze: The surface plasmon resonance in free and embedded Ag-clusters in the size range $1,5 \mathrm{~nm}<\mathrm{D}<$ 30 nm, Cryst. Res. Technol. 33, 1085 (1998) 185, 187

38. J. Gersten, A. Nitzan: Electromagnetic theory of enhanced Raman scattering by molecules adsorbed on rough surfaces, J. Chem. Phys. 73, 3023 (1980) 185

39. M. Weber, D. L. Mills: Interaction of electromagnetic waves with periodic gratings: Enhanced fields and the reflectivity, Phys. Rev. B 27, 2698 (1983) 185

40. F. J. García-Vidal, J. B. Pendry: Electromagnetic interactions with rough metal surfaces, Prog. Surf. Sci. 50, 55 (1995) 185

41. S. J. Oldenburg, R. D. Averitt, S. L. Westcott, N. J. Halas: Nanoengineering of optical resonances, Chem. Phys. Lett. 288, 243 (1998) 185

42. F. J. García-Vidal, J. B. Pendry: Collective theory for surface enhanced Raman scattering, Phys. Rev. Lett. 77, 1163 (1996) 185

43. M. Kahl, E. Voges, S. Kostrewa, C. Viets, W. Hill: Periodically structured metallic substrates for SERS, Sens. Actuators B 51, 285 (1998) 185

44. M. Kahl, E. Voges: Analysis of plasmon resonance and surface-enhanced Raman scattering on periodic silver structures, Phys. Rev. B 61, 14078 (2000) 185

45. R. Fuchs: Theory of the optical properties of ionic crystal cubes, Phys. Rev. B 11, 1732 (1975) 185 
46. W.-H. Yang, G. C. Schatz, R. P. van Duyne: Discrete dipole approximation for calculating extinction and Raman intensities for small particles with arbitrary shape, J. Chem. Phys. 103, 869 (1995) 185, 188

47. T. R. Jensen, G. C. Schatz, R.P. van Duyne: Nanosphere lithography: surface plasom resonance spectrum of a periodic array of silver nanoparticles by ultraviolett-visible extinction spectroscopy and electrodynamic modeling, J. Phys. Chem. B 103, 2394 (1999) 185, 188

48. N. Félidj, J. Aubard, G. Lévi: Discrete dipole approximation for ultravioletvisible extinction spectra simulation of silver and gold colloids, J. Chem. Phys. 111, 1195 (1999) 185, 188

49. J. P. Kottmann, O. J. F. Martin: Accurate solution of the volume integral equation for high permittivity scatterers, IEEE Trans. Antennas Propag. 48, 1719 (2000) 185, 189, 190

50. J. P. Kottmann, O. J. F. Martin, D. R. Smith, S. Schultz: Dramatic localized electromagnetic enhancement in plasmon resonant nanowires, Chem. Phys. Lett. 341, 1 (2001) 185, 186, 198

51. J.P. Kottmann, O. J. F. Martin, D. R. Smith, S. Schultz: Non-regularly shaped plasmon resonant nanoparticle as localized light source for near-field microscopy, J. Microscopy 202, 60 (2001) 185, 195, 200, 201

52. J. P. Kottmann, O. J. F. Martin, D. R. Smith, S. Schultz: Plasmon resonances of silver nanowires with a non-regular cross-section, Phys. Rev. B 64, 235402 (2001) 185, 190

53. J.P. Kottmann, O. J.F. Martin: Influence of the cross section and the permittivity on the plasmon-resonance spectrum of silver nanowires, Appl. Phys. B 73, 299 (2001) 185, 202

54. J. P. Kottmann, O. J. F. Martin, D. R. Smith, S. Schultz: Spectral response of Silver nanoparticles, Optics Express 6, 213 (2000) 185, 192, 195, 198

55. J. P. Kottmann, O. J. F. Martin, D. R. Smith, S. Schultz: Field polarization and polarization charge distributions in plasmon resonant particles, New J. Phys. 2, 27.1 (2000) 185, 192, 193, 195

56. J.P. Kottmann, O. J.F. Martin: Plasmon resonant coupling in metallic nanowires, Optics Express 8, 655 (2001) 185, 195

57. J.P. Kottmann, O. J. F. Martin: Retardation-induced plasmon resonances in coupled nanoparticles, Opt. Lett. 26, 1096 (2001) 185, 195

58. C. F. Bohren, D. R. Huffman: Absorption and Scattering of Light by Small Particles (Wiley, New York 1983) 186, 188, 189, 201

59. U. Kreibig, M. Vollmer: Optical Poperties of Metal Clusters, Springer Ser. Mater. Sci. 25 (Springer-Verlag, Berlin, Heidelberg 1995) 186, 187, 201

60. P. B. Johnson, R. W. Christy: Optical constants of the noble metals, Phys. Rev. B 6, 4370 (1972) 186, 187

61. R. Ruppin: Spherical and cylindrical surface polaritons in solids, in A. D. Boardman (Ed.): Electromagnetic Surface Modes (Wiley, Chichester 1982) 186

62. U. Kreibig, C. von Fragstein: The limitation of electron mean free path in small silver particles, Z. Physik 224, 307 (1969) 187

63. L. Genzel, T. P. Martin, U. Kreibig: Dielectric function and plasma resonances of small metal particles, Z. Physik B 21, 339 (1975) 187, 201

64. J.-Y. Bigot, V. Halté, J. C. Merle, A. Daunois: Electron dynamics in metallic nanoparticles, Chem. Phys. 251, 181 (2000) 187, 201

65. M. Brack: The physics of simple metal clusters: self-consistent jellium model and semiclassical approaches, Rev. Mod. Phys. 65, 677 (1993) 187, 201 
66. V. V. Kresin: Collective resonances and response properties of electrons in metal clusters, Phys. Rep. 220, 1 (1992) 187

67. V. Bonacic-Koutecky, P. Fantucci, J. Koutecky: Quantum-chemistry of small clusters of elements of group-IA, group-IB, and group-IIA: Fundamental concepts, predictions and interpretation of experiments, Chem. Rev. 91, 1035 (1991) 187, 201

68. O. J.F. Martin, N.B. Piller: Electromagnetic scattering in polarizable backgrounds, Phys. Rev. E 58, 3909 (1998) 188

69. M. Paulus, P. Gay-Balmaz, O. J. F. Martin: Accurate and efficient computation of the Green's tensor for stratified media, Phys. Rev. E 62, 5797 (2000) 188

70. M. Paulus, O. J. F. Martin: Light propagation and scattering in stratified media: A Green's tensor approach, J. Opt. Soc. Am. A 18, 854 (2001) 188

71. A. Taflove: Computational Electrodynamics, the FDTD Method (Artech House, Boston 1995) 188

72. B. T. Draine, P. J. Flatau: Discrete-dipole approximation for scattering calculations, J. Opt. Soc. Am. A 11, 1491 (1994) 188

73. P. J. Flatau: Improvements in the discrete-dipole approximation method of computing scattering and absorption, Opt. Lett. 22, 1205 (1997) 188

74. M. I. Stockmann, V.M. Shalaev, M. Moskovits, R. Botet, T.F. George: Enhanced Raman scattering by fractal clusters: Scale-invariant theory, Phys. Rev. B 46, 2821 (1992) 188

75. N. B. Piller, O. J. F. Martin: Increasing the performances of the coupled-dipole approximation: A spectral approach, IEEE Trans. Antennas Propag. 46, 1126 (1998) 188

76. J. van Bladel: Singular Electromagnetic Fields and Sources (Clarendon, Oxford 1991) 190, 195

77. L. D. Landau, E. M. Lifshitz, L.P. Pitaevskii: Electrodynamics of continuous media, Vol. 8 of Landau and Lifshitz course on theoretical physics, 2nd. ed. (Butterworth Heinemann, Oxford 1998) 193

78. B. Hecht, H. Bielefeld, Y. Inouye, D. W. Pohl, L. Novotny: Facts and artifacts in near-field optical microscopy, J. Appl. Phys. 81, 2492 (1997) 195

79. R. P. van Duyne: private communication 196

80. M. D. Malinsky, L. Kelly, G. C. Schatz, R. P. van Duyne: Chain length dependance and sensing capabilities of the localized surface plasmon resonance of silver nanoparticles chemically modified with Alkanethiol self-assembled monolayers, J. Am. Chem. Soc. 123, 1471 (2001) 196

81. A. Campion, P. Kambhampati: Surface-enhanced Raman scattering, Chem. Soc. Rev. 27, 241 (1998) 197

82. P. Gadenne, X. Quelin, S. Ducourtieux, S. Gresillon, L. Aigouy, J.-C. Rivoal, V. Shalaev, A. Sarychev: Direct observation of locally enhanced electromagnetic fields, Physica B 279, 52 (2000) 198

83. Y. C. Martin, H. K. Wickramasinghe: Resolution test for apertureless near-field optical microscopy, J. Appl. Phys. 91, 3363 (2002) 200

84. U. Kreibig, C. von Fragstein: Electronic properties of small silver particles: the optical constants and their temperature dependence, J. Phys. F. 4, 999 (1974) 201

85. N. D. Fatti, F. Vallée, C. Flytzanis, Y. Hamanaka, A. Nakamura: Electron dynamics and surface plasmon resonance nonlinearities in metal nanoparticles, Chem. Phys. 251, 215 (2000) 201 
86. Optical Society of America, in M. Bass (Ed.): Handbook of Optics, Vol. II., 2nd ed. (McGraw Hill, New York 1995) 203

87. H. Xu, J. Aizpurua, M. Käll, P. Apell: Electromagnetic contributions to singlemolecule sensitivity in surface-enhanced Raman scattering, Phys. Rev. E 62, 4318 (2000) 203 


\section{Index}

dispersion relation, 186, 201

electromagnetic enhancement, 197

Green's tensor, 188

- singularity, 189

nanoparticle fabrication, 185

nanophotonics, 184

nanowires, 183

particle background, 202

plasma frequency, 186

plasmon resonances

- distance dependence, 196

- electrostatic model, 185

- field distribution, 193, 195

- movies, 185

- near-field, 196

- non-regular particles, 186, 190

- numerical simulations, 185

- resonance width, 191
- scattering cross-section, 191

- scattering problem, 187

- shape effects, 191

- size effects, 191

- sphere, 186

polarizable background, 202

polarization charge distributions, 192, 193, 195

Raman enhancement, 184, 196, 197

scanning near-field optical microscope (SNOM), 195

scattering cross-section, 190

surface modes, 186

surface-enhanced Raman scattering

(SERS), 184, 196, 199

triangular particle, 191

volume integral equation, 188 